\title{
Sand Table Practice in Firemen's Psychological Correction Work in Remote Areas
}

\section{Bu Pengcui}

School of Education, Longdong University, Qingyang, China

\section{Email address:}

107691699@qq.com

\section{To cite this article:}

Bu Pengcui. Sand Table Practice in Firemen's Psychological Correction Work in Remote Areas. Science Innovation. Vol. 5, No. 5, 2017, pp. 303-306. doi: 10.11648/j.si.20170505.21

Received: June 30, 2017; Accepted: July 20, 2017; Published: August 16, 2017

\begin{abstract}
Through the survey of SCL-90 of 9 fire squadron firefighters team within 3 months of the Qingyang Pingliang area, Gansu province China area, Baiyin area etc. select the total score, number of positive items and the average scores of positive items were higher in the 22 students, were randomly divided into two groups, conducted 4 Sandplay intervention, intervention time is about an hour. At the end of the treatment, feedback reports are given to advise on several treatment options. Results: compared to firefighters who did not participate in Sandplay therapy, the descriptive statistics showed that all the factors in the intervention group scored lower after 1 months of intervention. In the $\mathrm{T}$ test, the total score, the number of positive items and anxiety factors were significantly different $(\mathrm{p}<0.01)$. Sand table themes vary dramatically from trauma to healing. Conclusion: the progress made by firefighters in sand table can be transferred to real life and has positive effects.
\end{abstract}

Keywords: Remote Area, Firefighters, Psychological Correction, Sandplay Therapy

\section{偏远地区消防员心理矫治工作中的沙盘实践}

\section{卜鹏翠}

教育学院, 陇东学院, 庆阳, 中国

\section{邮箱}

107691699@qq.com

摘要: 通过对中国甘肃省平凉地区、庆阳地区、白银地区等 9 个消防中队入队 3 个月内的消防员进行SCL-90的前期普测, 选出总分、阳性项目数和阳性项目均分均较高的学员 22 人, 随机分组, 分别进行了 4 次沙盘干预, 每次干预时间约为一 个小时。治疗结束后, 给予反馈报告, 提供若干矫治方式上的建议。结果：相比较于未参与沙盘治疗的消防员，从描 述性统计指标来看, 干预组消防员所有的因子在 1 个多月的干预后得分都有所降低。在 $\mathrm{T}$ 检验中, 总分、阳性项目数、 焦虑因子的前后测达到显著差异（ $\mathrm{p}<0.01 ）$ 。沙盘主题从“创伤”到“治愈”的变化显著。结论：消防员在沙盘中获得的 进步顺利迁移到实际生活中并存在积极的效果。

关键词：偏远地区，消防员，心理矫治，沙盘疗法

\section{1. 引言}

沙盘的应用领域在逐步扩大。[1]偏远地区的消防部队 除担负着繁重的灭火和社会抢险救援任务外, 还要担负偏
远城镇居民人身与财产安全保卫工作，偏远城市消防员的 职业具有环境复杂、条件艰苦、强制性强、机动性大、危 险性大等特点, 其职业的特殊性要求消防员必须具有过硬 的心理素质。那么, 借助完备的心理咨询工具实施心理矫 
治, 缓解消防员心理压力, 从而确保全体消防官兵思想稳 定就显得极其重要。[2]

\section{2. 研究目的与假设}

根据偏远地区消防场所的条件, 并基于现实心理矫治 工作的需要, 在分析心理学理论指导下, 浓缩沙盘疗法的 步骤和周期, 在消防员中尝试沙盘治疗方法, 并尝试探索 该疗法对消防员解除焦虑，应对突发性危机的实际效果。

随着治疗的进行, 消防员的心理健康状况有所改善。 消防员沙盘作品中创伤主题逐渐减少, 治愈主题逐渐增多。 消防员在沙盘中获得的进步顺利迁移到矫治生活中。[3]

\section{1. 研究设计}

等组前后测实验设计。通过干预组与控制组比较, 考 察沙盘疗法对干预组学员的心理状况的改善效果。

\section{2. 研究对象}

通过对中国甘肃省平凉地区、庆阳地区、白银地区等 9 个消防中队入队 3 个月内的消防员进行 SCL- 90 的前期普 测, 并挑选出总分、阳性项目数、阳性项目均分得分均较 高的学员 22 人, 随机分成干预组和控制组。

\section{3. 研究工具}

（1）症状自评量表（SCL-90）

（2）沙盘治疗工具: 沙盘 4 个, 沙具 2556 个, 笔, 数 码相机, 记录表等。

\section{4. 研究过程}

\subsection{1. 研究对象的选择}

通过SCL-90对入队 3 个月内的消防员进行 SCL-90的 前期普测, 挑选出总分、阳性项目数、阳性项目均分和大 多数因子得分均较高的学员 22 人, 随机分成干预组和控制 组。

\section{4.2. 半结构化访谈}

与消防员的访谈定在实施初次沙盘治疗前, 时间为 30 分钟, 内容包括: 消防员基本人口学信息; 消防员个人成 长经历及家庭情况; 描述一下消防员在社会上的交友相处 的情况; 消防员的救火史, 火场经历; 对自我心理健康状 态的认知。

\subsection{3. 沙盘疗法的流程}

沙盘疗法的本质是非语言的无意识治疗, 因此在进行 过程中, 咨询师除了无条件的关注和包容之外, 陪同一起 度过这些制作沙盘的时间是十分重要的, 实际上心理咨询 师在旁边的投入和关注、和消防员对于沙盘的共同欣赏、 和消防员的简短对话, 都会无意识中深深的影响消防员的 无意识, 进而产生治疗功效。这些, 正是沙盘疗法的关键 点。沙盘疗法的流程包括三个阶段： [4]

(1) 导入
本次研究中, 初始导入的指导语为: “我们要进行一 个小游戏, 这里有一个装有沙子的小箱子, 架子上放有很 多型具, 请你用沙子和型具, 在箱子里摆放出自己想像的 空间或人物造型, 摆放什么都可以。”很多消防员在做初 始沙盘的时候会出现紧张或忙乱的情形, 此时可以使用下 面的指导语: “你先试着用手摸摸沙子, 不要着急, 感受 一下沙子, 看会有什么感觉, 随着这种感觉, 到边上的架 子上寻找自己想要的玩具模型, 放在沙盘里。”

在对这 4 个学员的实际操作过程中, 有 2 个学员在进行 补充指导语后, 摸了沙子仍然处于比较茫然的状态, 笔者 就又补充了一句: “你们可以先站起来, 看看架子上的玩 具, 感受一下, 然后再选。”对于初始阶段的紧张焦虑, 特别需要予以重视, 根据笔者的案例经验, 就沙盘疗法而 言, 初始导入的成功往往标志着咨询的成功。

(2) 分析

体验沙盘, 理解沙盘是这一环节的关键。体验沙盘的 阶段不是一种令人䀟尤的沉默, 而是一种有意义的沉默关 注。咨询师和消防员都将注意指向消防员内心深处，因而 有了更深刻的理解。在消防员静下心来体验自己的世界时, 咨询师不加以任何评价, 要以尊重、欣赏的态度来看待这 个世界, 无条件关注和接纳这个世界的诞生, 即使它是如 此的紧张和不安, 但这就是消防员的真实精神世界, 是他 们演绎的一段与自己紧张而高焦虑的工作相适应的鲜活 的心路历程, [5]

因此体验过程中消防员流露出的任何情绪表现都要 加以关注。但是这个时候最好不要做出任何的情感方面的 解释, 也不要提出任何的建议, 也不要提任何的问题干预 他们的体验。沙盘的体验阶段单纯是学员与其自己的内心 世界进行对话的过程, 咨询师需要坚持静默关注的态度, 保护着并关注着。体验之后的询问也不需太多, 良性互动 并助其领悟。这一过程对于咨询师的素质和理论功底要求 比较高, 否则不是陷入咒长的问题反复中, 便是导致整体 的失败。[6]

(3) 转化

无意识以及无意识的意向, 都是具有生命意义的存在, 具有心性的自主性。[7]心理分析对无意识的尊重, 包含着 这样一种思想和观点, 与自我心性积极感受、接触和沟通, 从中获得启发和力量, 获得内在治愈和发展, 转化与超越, 获得心理与人格的整合, 是沙盘治疗的根本目的和终极目 标。[8]

\section{3. 沙盘疗法实验结果}

\section{1. SCL-90 前测和后测实验差异比较}

\section{1. 1. 控制组SCL-90 前测和后测比较}

在控制组消防员的SCL-90前后测分数比较中发现, 他 们在各因子分上都没有出现显著差异。( T检验结果显示, $\mathrm{P}$ 值均大于 0.05 )

\section{1. 2. 干预组SCL-90 前测和后测比较}

比较于控制组消防员，进行了沙盘疗法的消防员在 SCL-90问卷前后测分数上表现出了差异。从描述性统计指 
标来看, 所有的因子在 3 个多月的沙盘疗法干预后得分都 有所降低。在 $\mathrm{T}$ 检验中, 总分、阳性项目数、抑郁和焦虑 因子的前后测达到显著差异 $(\mathrm{p}<0.01)$; 而阳性项目均分、 躯体化、人际关系、敌对、偏执因子的前后测也有差异 $(p<0.05) 。 （$ 详见下表1）

表1 干预组SCL-90前测后测得分比较。

\begin{tabular}{lllll}
\hline 因子 & 前测 & 后测 & t & $\mathbf{P}$ \\
\hline 总分 & $181.70 \pm 5.54$ & $150.17 \pm 19.29$ & 4.850 & $0.005^{* *}$ \\
阳性项目数 & $62.83 \pm 4.45$ & $50.28 \pm 8.54$ & 4.528 & $0.006^{* *}$ \\
阳性项目均分 & $2.28 \pm 0.18$ & $2.02 \pm 0.20$ & 3.206 & $0.027^{*}$ \\
躯体化 & $2.15 \pm 0.46$ & $1.66 \pm 0.51$ & 3.195 & $0.024^{*}$ \\
强迫症状 & $2.00 \pm 0.44$ & $1.85 \pm 0.27$ & 2.074 & 0.093 \\
人际关系 & $2.12 \pm 0.25$ & $1.75 \pm 0.41$ & 3.164 & $0.024^{*}$ \\
抑郁 & $2.17 \pm 0.18$ & $1.47 \pm 0.41$ & 3.970 & $0.008^{* *}$ \\
焦虑 & $2.27 \pm 0.19$ & $1.64 \pm 0.30$ & 3.845 & $0.009^{* *}$ \\
敌对 & $2.13 \pm 0.47$ & $1.68 \pm 0.24$ & 2.530 & $0.044^{*}$ \\
恐怖 & $1.91 \pm 0.48$ & $1.58 \pm 0.42$ & 2.517 & $0.046^{*}$ \\
偏执 & $2.16 \pm 0.16$ & $1.81 \pm 0.26$ & 2.655 & $0.045^{*}$ \\
精神病性 & $2.05 \pm 0.35$ & $1.96 \pm 0.22$ & 1.616 & 0.167 \\
其他 & $2.31 \pm 0.40$ & $2.03 \pm 0.26$ & 2.181 & 0.081 \\
\hline
\end{tabular}

\section{2. 主题转化分析}

沙盘疗法强调在无意识水平的工作, 主要通过象征性 的语言来达到表现和沟通的目的。沙盘的主题是对每次治 疗所表现出的象征性意义的总结。沙盘中出现的系列意象 会构成一些基本的主题。米歇尔曾归纳了两大基本主题: 创伤主题和治愈主题。创伤主题包括混乱、空洞、分裂、 限制、忽视、隐藏、威胁、受伤等, 治愈主题则包括联结、 旅程、深入、新生、趋中、灵性等。在沙盘治疗进程中, 主题会出现一些变化, 治愈的主题会逐渐增多, 创伤的主 题会逐渐减少。[9]

\section{4. 研究结果分析}

\section{1. 咨询师无条件地接纳为消防员营造了一个自由受保 护的氛围}

心理健康指导尽可能的为学员创造相对正面的矫治 环境, 和正向引导的咨询服务, 消防员在制作沙盘作品的 过程中, 研究者不会打扰他们的创作过程, 只是让他感受 到他正在被积极地关注着, 并在适当的情况给予帮助, 此 时, 他们把潜意识里的内容逐渐展现出来, 焦虑等负性情 绪得到了疏导, 同时也感受到了关注, [9]这种咨访关系本 身对消防员的身心发展具有积极的影响。

\section{2. 沙盘疗法的自愈作用效果明显}

在沙盘疗法的过程中, 笔者除了一开始的引导, 几乎 不做任何的干预与评价, 一切都是由消防员自发完成的。 第一阶段, 沙盘的画面里有战斗机、植物、动物, 场景比 较杂乱, 是比较肤浅的, 仅仅是装饰性的。同时, 出现一 些创伤主题, 如发起战争、受伤或死亡等等, 第二阶段, 矛盾冲突逐渐升级, 消防员显得比较兴奋, 此时他们正在 宣泄内心的负性情绪, 但是也会消耗内心的能量, 慢慢地 会产生疲倦感。第三阶段, 一些治愈主题出现了, 攻击性
的模型逐渐减少, 正面的力量慢慢强大起来, 画面出现了 一些扭转, 正义的一方赢得了战争或者战争双方出现了妥 协, 象征能量的水源与植物的出现, 新生命的诞生, 人物 复活等等, 此时内心的负性能量转换为了正面的积极能量。 第四阶段, 画面回归到现实生活的平静, 场景中出现了具 有现实意义的房子、人和动物, 在这个时候消防员的心境 往往比较宁静愉悦, 作品中的治愈主题居多, 甚至有位消 防员全部都是治愈主题, 没有创伤主题。[10]

\section{3. 沙盘治疗的经历与体验得到迁移}

根据消防员的评价, 战友间的交往比以前更加顺畅, 紧张焦虑, 人际关系敏感, 应激障碍等消极心理问题慢慢 减少, 消防战士逐渐懂得如何更好地控制情绪和解决突发 问题。

\section{5. 结论}

沙盘疗法能够有效检测消防员的心理健康状态。采用 SCL-90对参加沙盘疗法的消防员的测试结果显示, 在总分、 阳性项目数、躯体化和焦虑等因子上均具有比较显著的效 果; 主题从“创伤”到“治愈”的变化显著。参加沙盘疗法的 消防员的沙盘作品出现战争、灾难等创伤主题逐渐减少, 和平、安全等治愈主题逐渐增多的趋势, 且初始作品的创 伤主题和治愈主题的数量与最后一次作品的数量的差异 达到显著性水平;

根据治疗前后指导员与中队长的评价比较, 发现消防 员在情绪控制、环境适应与自我调节等方面有了一定的改 善, 说明沙盘疗法的效果被迁移到消防员的日常矫治生活 中。

\section{致谢}

本文为甘肃省“十二五”教育科学规划课题(编号: GS[2015]GHB0958)的阶段性成果之一。

\section{参考文献}

[1] Carey. Lois, Short-term family sandplay therapy. Short-term play therapy for children [M], Newyork, NY, US: Guilford Press, 2016: 202-215.

[2] 申荷永, 陈㑆, 高岗. 沙盘游戏治疗的历史与理论 $[\mathrm{J}]$. 心 理发展与教育, 2005, 2: 126-128。

[3] 肖春红. 沙盘游戏疗法的应用及研究进展 $[\mathrm{J}]$. 中国疗养医 学, 2013, (07): 609-611。

[4] 陈顺森. 沙盘疗法缓解初中生考试焦虑有效性的研究 [D], 河北大学, 2005。

[5] 马建军. 现实疗法对劳教人员心理矫治效果的实验研究 [D]. 湖南师范大学, 2004。 
[6] 耿进苏，关于团体沙盘游戏技术干预自考生考试焦虑效果 的研究 $[D]$, 华东师范大学, 2008。

[7] 陈顺森. 箱庭疗法在学校心理咨询中的应用 [J]. 漳州师范 学院学报, 2006, (3): 142-143。

[8] 张雯，刘亚茵，张日昇，团体箱庭疗法对人际交往不良大 学生的治疗过程与效果研究 $[\mathrm{J}]$. Chinese Journal of Clinical Psychology, 2010, (2): 264-266。
[9] 宋斌. 沙盘游戏主题与自我防御机制的心理分析研究 [A]. 中国心理卫生协会大学生心理咨询专业委员会. 中国 特色大学生心理健康教育—第十二届全国大学生心理健 康教育与咨询学术交流会论文集 $[\mathrm{C}]$. 中国心理卫生协会大 学生心理咨询专业委员会, 2015: 5。

[10] 刘路. 沙盘游戏对高校学生的干预研究综述 [J]. 新疆广播 电视大学学报, 2015, (03): 67-71。 